\title{
Choosing the road less traveled by: a ligand-receptor system that controls target recognition by Drosophila motor axons
}

\author{
Kai Zinn ${ }^{1}$ \\ Division of Biology, California Institute of Technology, Pasadena, California 91125, USA
}

In this issue of Genes \& Development, Siebert and colleagues (pp. 1052-1062) define a ligand-receptor system that controls motor axon guidance and target recognition in the Drosophila embryo. The beaten path (beat) and sidestep (side) genes were known to be important regulators of motor axon guidance. Siebert and colleagues now show that Beat and Side are cell surface proteins that physically interact with each other, and that Beatexpressing motor axon growth cones reach their targets via recognition of Side-expressing pathways.

The Drosophila neuromuscular system has provided an ideal experimental arena in which to explore the genetic basis of neural circuit formation. Its virtues include simplicity, invariance, and visibility. In abdominal segments A2-A7, there are $\sim 36$ motor neurons per fundamental unit, and these form synapses in an invariant manner on 30 individually identifiable muscle fibers (Keshishian et al. 1996; Landgraf and Thor 2006). Each axon and synapse in this system can be visualized in live embryos and larvae expressing green fluorescent protein (GFP) markers, as well as in dissected "fillet" preparations stained with antibodies such as the motor axon-specific monoclonal antibody (mAb) 1D4 (Vactor et al. 1993; Zito et al. 1999; Kraut et al. 2001).

Motor axons follow defined pathways out of the CNS to reach the vicinity of their muscle targets. Axons of sensory neurons form segments of the pathways followed by "pioneer" motor axons, and later motor axons grow along these pioneers. Many cell surface receptors and secreted guidance proteins have been characterized that affect the selection of pathways by motor axons. However, we still know relatively little about the molecular mechanisms by which motor axon growth cones identify intermediate targets such as sensory axons. We also do

[Keywords: Drosophila; motor axon guidance; Sidestep; Beaten path substrate pathway; in vivo imaging; time-lapse movie]

${ }^{1}$ Correspondence.

E-MAIL zinnk@caltech.edu; FAX (626) 449-0679.

Article published online ahead of print. Article and publication date are online at http://www.genesdev.org/cgi/doi/10.1101/gad.1803009. not understand why motor axons later decide to stop growing along these axonal pathways and instead turn into the appropriate muscle field. In this issue of Genes \& Development, a study by Aberle and colleagues (Siebert et al. 2009/ provides important new insights into these processes. Siebert et al. (2009) identify a ligand-receptor system that controls both the selection of intermediate axonal targets and the later decision to leave these targets and navigate into the muscle fields.

\section{The history of Beat and Side}

This story begins with the pioneering anatomical screens for axon guidance phenotypes conducted in Corey Goodman's laboratory in the 1990s (Vactor et al. 1993; Fambrough and Goodman 1996; Sink et al. 2001). Two mutations isolated in screens of embryo collections stained for motor axons using mAb 1D4-beaten path (beat) and sidestep (side)-produced similar phenotypes in which motor axons failed to turn into the muscle fields and innervate their targets, instead remaining trapped within their axon bundles (Vactor et al. 1993; Fambrough and Goodman 1996; Sink et al. 2001). In the most extreme version of the beat and side phenotypes, the motor axon network was reduced to two bundles: the intersegmental nerve (ISN) and the segmental nerve (SN). This is the same phenotype that is caused by complete genetic ablation of the muscles (Landgraf et al. 1999).

When beat and side were cloned, it was found that both genes encode proteins with extracellular regions containing immunoglobulin-like (Ig) domains (Fambrough and Goodman 1996; Sink et al. 2001). Beat is expressed on motor axons, while Side is expressed on sensory axons and muscles in the periphery. It was also observed that ectopic expression of Side on tracheal branches caused motor axons to grow abnormally on the tracheae in preference to their normal pathways. Since Side protein has a transmembrane domain, this led to the hypothesis that Side is a target recognition receptor that attracts motor axons (Sink et al. 2001). This model is confirmed and extended by the study by Siebert et al. (2009). 
The initial interpretation of the beat phenotype was different. Beat was thought to be a secreted protein, and it was found that the beat phenotype can be suppressed by reducing the levels of the homophilic cell adhesion molecule Fasciclin II (FasII), which is expressed by all motor axons. Based on these findings, Beat was proposed to be an "anti-adhesion" factor whose expression by motor axons allows them to defasciculate from their axon bundles at the sites where they turn into their target muscle fields. This defasciculation is an essential prerequisite to the selection of muscle fibers as the preferred substrate for growth (Fambrough and Goodman 1996).

\section{Beat and Side define a ligand-receptor system that controls axon guidance}

Siebert et al. (2009) now provide a direct linkage between Beat and Side, demonstrating that Beat is actually a cell surface protein that is a neuronal receptor (or a component of a receptor) for Side. This means that the beat and side phenotypes now can be understood as defects in a single mechanism. The new study by Siebert et al. (2009) also employs genetic evidence to support an aesthetically pleasing model for motor axon guidance and target recognition, in which Beat-expressing axons are attracted to their intermediate targets via recognition of Side. After the axons contact an intermediate target, Side is down-regulated there and up-regulated on the next target. In this manner, motor axons are able to navigate from the CNS to the muscle fibers by following a continuous "breadcrumb trail" of Side (see Fig. 7 of Siebert et al. 2009 for a diagram of the model).

The new study by Siebert et al. (2009) begins with a detailed analysis of the Side protein expression pattern. As motor axons leave the ventrally located CNS during mid-embryogenesis, they extend dorsally along the edges of a triangular patch of Side-expressing cells, with ISN axons growing on the anterior edge of the patch and SN axons choosing the posterior edge. Next, Side is turned off in this patch and turned on in dorsal sensory neurons whose axons form an intermediate target for the ISN motor axons that will innervate dorsal muscle fibers. Finally, at the time at which ISN axons leave the sensory pathway and turn into the muscle field, Side is downregulated on sensory axons and up-regulated on muscle fibers.

Live imaging of motor axons in wild-type embryos showed that when the ISN axon bundle is growing along the sensory axon substrate it has a single, rapidly moving growth cone. In side mutants, however, the ISN has multiple complex growth cones that move more slowly. These sometimes still reach the dorsal regions of the periphery, suggesting that other cues can be used for guidance, but recognition of their pathways is clearly impaired in the absence of Side. In segments of side animals in which the ISN fails to reach dorsal regions, the neuromuscular junctions (NMJs) on dorsal muscles 1 and 9 do not form, and this phenotype persists through larval development.
If Side is precociously expressed on muscle fibers while motor axons are still growing along the sensory axon substrate, guidance is also impaired. In this case, ISN motor axons leave the sensory pathway and diverge into inappropriate muscle fields that are ventral to their normal targets. As a consequence, all of the dorsal muscles sometimes fail to be innervated in larvae. Ectopic expression of Side can also divert motor axons to targets that they normally never recognize. Expression of Side on the tracheae causes ISN axons to grow along tracheal branches (Sink et al. 2001), while expression on hemocytes can cause growth cones to attach to these cells and remain stuck there.

Loss of Beat produces the same loss of dorsal NMJs as loss of Side, and with a similar penetrance. This allowed Siebert et al. (2009) to examine genetically whether Beat and Side are likely to be components of the same mechanism. If Side is expressed precociously on muscles, muscles 1 and 9 are almost never innervated. If Beat is removed genetically from the motor axons of these animals, however, this phenotype is suppressed, so that the dorsal muscles lack innervation in only $\sim 25 \%$ of segments, which is the same penetrance seen in beat mutants. This shows that motor axons cannot sense ectopically expressed Side if they lack Beat. (The fact that muscles 1 and 9 are successfully innervated $\sim 75 \%$ of the time in beat and side mutants presumably indicates that motor axons can use other, as yet unidentified, cues to reach their targets if Side is not present.)

Having shown that Beat is necessary for reception of the Side signal, Siebert et al. (2009) next examined whether Beat and Side can physically interact. To do this, they used the Drosophila Schneider 2 (S2) cell aggregation assay, in which normally nonadherent S2 cells form clumps if they express cell adhesion molecules. S2 cells expressing Beat or Side alone do not aggregate, but when cells expressing Beat and one fluorophore are mixed with cells expressing Side and a different fluorophore, bicolored aggregates containing both cell types are observed. Beat and Side can also be coimmunoprecipitated from S2 cells, providing additional evidence that they interact. The study by Siebert et al. (2009) does not prove that Beat and Side directly bind to each other, but this is the simplest hypothesis to explain the data.

\section{Signaling consequences of engagement between Beat and Side}

If both components of this heterophilic system are expressed together in one cell type (or come into contact on the wrong cells), signaling appears to be blocked. Siebert et al. (2009) show that expression of Beat and Side together on muscles neutralizes the effect of Side expression alone, allowing more ISN axons to innervate their normal targets. Muscle Beat expression alone may antagonize endogenous Side (on muscles or sensory axons), since it causes some innervation failures and axon guidance errors (Fambrough and Goodman 1996). Similarly, expression of Side on motor neurons also produces 
guidance phenotypes, possibly through blockage of Beat signaling (Sink et al. 2001).

More importantly, contact of Beat-expressing growth cones with Side-expressing sensory neurons causes downregulation of Side protein expression. This allows the creation of a trail that efficiently leads the axons to their targets, since the affinity of motor axon growth cones for the sensory axon intermediate target would be reduced once Side is turned down in sensory neurons. This would facilitate selection of the muscle substrate, which has turned on Side expression by this time, as the preferred substrate for motor axon growth. The down-regulation of Side expression by contact with Beat neurons may be different from the blockage of Side signaling that occurs when Beat and Side are expressed together, because Side is not down-regulated when Beat is expressed on muscles but not on motor neurons. If Beat was a secreted protein as proposed previously (Fambrough and Goodman 1996), one might have expected that expression on muscles adjacent to sensory neurons could produce the same effects as expression in motor neurons.

Perhaps cell surface Beat on motor axons interacts with an additional receptor that is normally expressed on sensory neurons together with Side, and signaling through that receptor causes down-regulation of Side expression. It remains to be determined whether Beat expression on sensory neurons alone would also fail to downregulate Side. If this was observed, it would suggest that heterophilic cell-cell interactions are required for downregulation.

\section{Future directions}

It will be of interest to examine the mechanisms by which contact between motor and sensory neurons down-regulates Side expression. We do not know if this down-regulation is at the level of transcription, translation, or protein stability. Screens could be performed to search for mutants in which Side fails to be downregulated, in order to identify the components of this putative signaling system.

The partial penetrance of the side and beat phenotypes implies the existence of other guidance and targeting cues that can substitute for this ligand-receptor system. The earlier studies showing that the beat phenotype can be partially suppressed by reducing interaxonal adhesion (Fambrough and Goodman 1996) show that the pathway decisions affected by Side and Beat are determined by relative rather than absolute preferences. When beat mutant axons adhere less tightly within a bundle, the alternative cues can direct them more easily into their target fields. Such cues might be identified by looking for mutations that increase the penetrance of side and beat.

Finally, the demonstration that Beat and Side physically interact opens the door to an examination of a complex network of interactions that may be important for neuronal circuit formation within the fly brain and ventral nerve cord. A study on "The Beat Generation" (Pipes et al. 2001) showed that there are at least 14 Beat family members in Drosophila, many of which are selectively expressed in subsets of neurons. It also provided evidence that some of the other Beats participate in axon guidance (Pipes et al. 2001).

Side is a member of a closely related family of eight Drosophila proteins. None of the Side paralogs have been characterized genetically. The finding that Side and Beat represent a ligand-receptor pair suggests that specific interactions between Side and Beat family members may be involved in wiring other circuits in the fly. One prediction can be made already, based on the results of Pipes et al. (2001). They showed that the transverse nerve (TN), which is formed by fasciculation of axons of the TMN (transverse motor nerve) with the axon of the peripheral LBD (lateral bipolar dendrite) neuron, is affected by mutations in beat Ic. In these mutants, the TMN and LBD axons often fail to come together. Since Beat Ic is only expressed in the CNS, these results suggest that a Side paralog that binds to Beat Ic should be expressed on the LBD axon, and that loss of this paralog should produce a beat Ic-like phenotype.

In summary, the results of Siebert et al. (2009) in this issue of Genes \& Development represent an important step forward in our understanding of axon targeting and neural circuit formation in Drosophila. Although vertebrates have many Ig domain proteins with structures similar to Beat and Side, these proteins do not have distinct vertebrate orthologs. Nevertheless, this type of mechanism still may represent a paradigm for axon guidance in vertebrate systems, since vertebrate axons also navigate to their final destinations via recognition of intermediate targets.

\section{References}

Fambrough, D. and Goodman, C.S. 1996. The Drosophila beaten path gene encodes a novel secreted protein that regulates defasciculation at motor axon choice points. Cell 87: 10491058.

Keshishian, H., Broadie, K., Chiba, A., and Bate, M. 1996. The Drosophila neuromuscular junction: A model system for studying synaptic development and function. Annu. Rev. Neurosci. 19: 545-575.

Kraut, R., Menon, K., and Zinn, K. 2001. A gain-of-function screen for genes controlling motor axon guidance and synaptogenesis in Drosophila. Curr. Biol. 11: 417-430.

Landgraf, M. and Thor, S. 2006. Development of Drosophila motoneurons: Specification and morphology. Semin. Cell Dev. Biol. 17: 3-11.

Landgraf, M., Baylies, M., and Bate, M. 1999. Muscle founder cells regulate defasciculation and targeting of motor axons in the Drosophila embryo. Curr. Biol. 9: 589-592.

Pipes, G.C., Lin, Q., Riley, S.E., and Goodman, C.S. 2001. The Beat generation: A multigene family encoding IgSF proteins related to the Beat axon guidance molecule in Drosophila. Development 128: 4545-4552.

Siebert, M., Banovic, D., Goellner, B., and Aberle, H. 2009. Drosophila motor axons recognize and follow a Sidesteplabeled substrate pathway to reach their target fields. Genes \& Dev. (this issue). doi: 10.1101/gad.520509.

Sink, H., Rehm, E.J., Richstone, L., Bulls, Y.M., and Goodman, C.S. 2001. sidestep encodes a target-derived attractant 
essential for motor axon guidance in Drosophila. Cell 105: $57-67$.

Vactor, D.V., Sink, H., Fambrough, D., Tsoo, R., and Goodman, C.S. 1993. Genes that control neuromuscular specificity in Drosophila. Cell 73: 1137-1153.

Zito, K., Parnas, D., Fetter, R.D., Isacoff, E.Y., and Goodman, C.S. 1999. Watching a synapse grow: Noninvasive confocal imaging of synaptic growth in Drosophila. Neuron 22: 719729. 


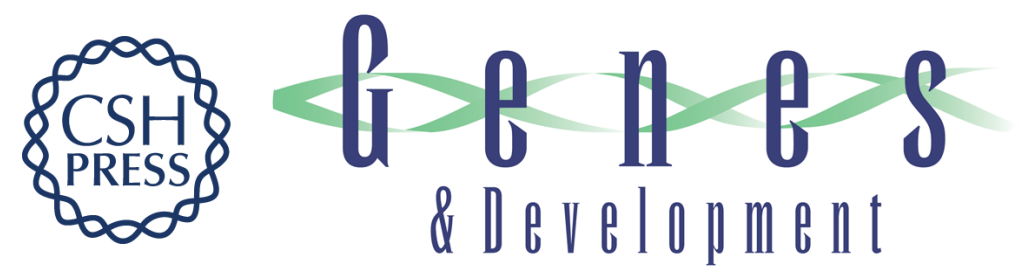

\section{Choosing the road less traveled by: a ligand-receptor system that controls target recognition by Drosophila motor axons}

Kai Zinn

Genes Dev. 2009, 23: originally published online April 15, 2009

Access the most recent version at doi:10.1101/gad.1803009

\section{Related Content \\ Drosophila motor axons recognize and follow a Sidestep-labeled substrate pathway to reach their target fields \\ Matthias Siebert, Daniel Banovic, Bernd Goellner, et al. \\ Genes Dev. May , 2009 23: 1052-1062 \\ References This article cites 10 articles, 2 of which can be accessed free at: \\ http://genesdev.cshlp.org/content/23/9/1042.full.html\#ref-list-1 \\ Articles cited in: \\ http://genesdev.cshlp.org/content/23/9/1042.full.html\#related-urls \\ License \\ Email Alerting \\ Receive free email alerts when new articles cite this article - sign up in the box at the top Service

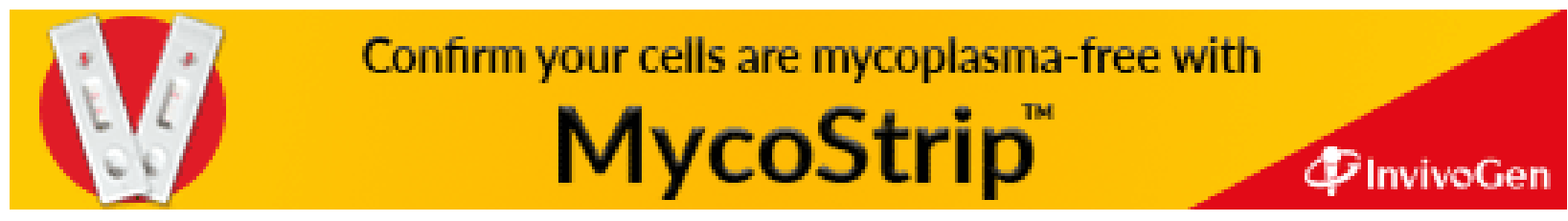

